\title{
MGPDI: modelo de gestión de la I+D+i en las organizaciones $^{1}$
}

Kival Weber ${ }^{2}$, José Antonio Antonioni ${ }^{3}$, Rosane Melchionna ${ }^{4}$, María Teresa Villalobos ${ }^{5}$, Ana Marcia Duarte $^{6}$, Juliana Herbert ${ }^{7}$, Cristina Machado ${ }^{8}$, Renato Machado ${ }^{9}$, Radamés Pereira ${ }^{10}$, Cristiano Schwening ${ }^{11}$

1. Situación a diciembre de 2016. Este artículo está basado en la ponencia presentada, como parte de la sección Call for Papers, durante el I Congreso Internacional de Ciencias de la Gestión: Gestión de la Innovación e Innovación en la Gestión, desarrollado del 29 de setiembre al 2 de octubre de 2015 en la Pontificia Universidad Católica del Perú por el Departamento Académico de Ciencias de la Gestión y la Facultad de Gestión y Alta Dirección.

2. Consultor Sénior en TIC, Calidad e Innovación - Curitiba-PR, Brasil - kival_weber@yahoo.com.br

3. SOFTSUL - Associação Sul-riograndense de Apoio ao Desenvolvimento de Software - Porto Alegre-RS, Brasil jaa@softsul.org.br

4. SOFTSUL - Associação Sul-riograndense de Apoio ao Desenvolvimento de Software - Porto Alegre-RS, Brasil rosane@softsul.org.br

5. PUCP - Pontificia Universidad Católica del Perú, Departamento de Ciencias - Sección Matemáticas, Especialidad Estadística - Lima, Perú - mtvillalobosa@pucp.edu.pe

6. UNOESC - Universidade do Oeste de Santa Catarina - Chapecó-SC, Brasil - ana.duarte@unoesc.edu.br

7. UFCSPA - Universidade Federal de Ciências da Saúde de Porto Alegre - Porto Alegre-RS, Brasil - julianash@ufcspa.edu.br / Facultad de Ingeniería, Universidad de la República, Programa de Desarrollo de las Ciencias Básicas (PEDECIBA) - Montevideo, Uruguay - jherbert@fing.edu.uy

8. CELEPAR - Companhia de Tecnologia da Informação e Comunicação do Paraná - Curitiba-PR, Brasil / QUALITYFOCUS Curitiba-PR, Brasil- cristina@pr.gov.br

9. QUALITYFOCUS - Curitiba-PR, Brasil - renato@qualityfocus.com.br

10. UNOCHAPECÓ - Universidade Comunitária da Região de Chapecó - Chapecó-SC, Brasil - radames@unochapeco.edu.br

11. ENGSOFT - Florianópolis-SC, Brasil-cristiano@engsoft.com.br 
Una estrategia para promover la innovación en las organizaciones consiste en la adopción de sistemas de gestión de la investigación, desarrollo e innovación $(I+D+i)$. El objetivo de este artículo es describir la situación actual del nuevo modelo de gestión de I+D+i (MGPDI) que es aplicable a cualquier organización, independientemente del tamaño, tipo y actividad. La novedad está en la definición del: 1) modelo de referencia MR-MGPDI, basado en buenas prácticas de innovación y en los requisitos de las normas ABNT NBR 16501 y AENOR UNE 166001-166002; y 2) modelo de evaluación MA-MGPDI, basado en la familia de normas ISO/IEC 33000 de evaluación de procesos. Hasta diciembre de 2016, hay tres objetivos: 1) desarrollar, probar y publicar dos guías MGPDI (Guía general y Guía de evaluación); 2) capacitar a los primeros consultores de implementación, evaluadores e instructores; y 3) realizar tres implementaciones y evaluaciones piloto del MGPDI en organizaciones del sur de Brasil, incluyendo el soporte de dos herramientas de software en línea (SGPDI y MGPDI AA). MGPDI es un modelo de proceso con un gran potencial de replicación en los países de América Latina y el Caribe.

Palabras clave: investigación, desarrollo, innovación, l+D+i, modelo de gestión

A strategy to promote innovation in organizations consists of the adoption of Research, Development and Innovation (R\&D\&l) management systems. This paper aims at describing the current situation of the new R\&D\&I Management Model (MGPDI) that is applicable to any organization, independently of the size, type and activity. The novelty is in the definition of the: 1) Process Reference Model MR-MGPDI based on innovation best practices and on requirements of the Brazilian ABNT NBR 16501 and the Spanish AENOR UNE 166001-166002 standards; 2) Process Assessment Model MA-MGPDI based on the family of standards ISO/IEC 33000 for process assessment. Until December 2016 there are three goals: 1) to develop, test and publish two MGPDI guides (General Guide and Assessment Guide); 2) train the first implementation consultants, assessors and instructors; 3) perform three pilot of MGPDI implementation and assessment in organizations in southern Brazil, including tests with two online software support tools (SGPDI and MGPDI AA). MGPDI is a process model with great potential of replication in Latin America and Caribbean countries.

Keywords: research, development, innovation, R\&D\&I, management model 


\section{Introducción}

Una tendencia en la gestión de negocios es el «abordaje bimodal», destacado en el Gartner Symposium/IT Expo del 8 de octubre de 2014 (Orlando, EEUU.) Para sobrevivir y prosperar, las organizaciones deben tener una parte sólida (modo sólido, como una roca) y una parte fluida (modo flujo continuo, como en el canotaje slalom). El modo "roca» está asociado a la gestión tradicional y tiene su enfoque en la búsqueda de la excelencia organizacional; por ejemplo, la mejora de procesos (Kalinowski y otros, 2015; Weber y otros, 2015b), resultando en una fuerte defensa (por analogía deportiva). El modo "canotaje slalom» está asociado a una gestión innovadora y tiene su enfoque en la fluidez, buscando ser fuerte también en el ataque (por analogía deportiva), liberando tiempo y recursos para la búsqueda de la innovación. Originalmente, este concepto estaba relacionado con el uso de métodos ágiles en la gestión del desarrollo de software, pero actualmente la ventaja del atacante está relacionada con la agilidad y a la fluidez en la gestión de negocios en las organizaciones de cualquier sector, con el uso de tecnologías capaces de procesar volúmenes extraordinarios de datos y con la búsqueda de la innovación como un diferencial competitivo (Charan, 2015).

Cada vez más, la innovación es esencial para que las organizaciones sobrevivan y prosperen. Básicamente, la innovación puede surgir de dos formas:

- Innovación cerrada, en la cual toda la I+D es hecha internamente buscando mejorar la competitividad de la organización en su mercado actual.

- Innovación abierta, en la cual la búsqueda de conocimientos (no apenas tecnologías) en la I+D es hecha tanto externa como internamente en la organización, buscando aumentar su competitividad, ya sea en el mercado actual o en nuevos negocios innovadores (Chesbrough, 2003).

El modelo de innovación cerrada todavía es usado, pero hoy prevalecen modelos de innovación abierta, tales como:

- Innovación lineal, sin realimentación, con destaque para modelos "science push» y "demand pull», como el modelo de la cadena de valor de la innovación (Hansen y Birkinshaw, 2007).

- Innovación basada en agrupaciones productivas locales y en clusters regionales, como el modelo Open Regional Innovation System (ORIS) (Belussi, Sammarra y Sedita, 2010).

- $\quad$ Sistemas de innovación basados en la triple hélice (Ranga y Etzkowitz, 2013).

- Innovación basada en sistemas de gestión de la investigación, desarrollo e innovación (I+D+i) (Ferreira y Cauchick-Miguel, 2013), como el modelo MGPDI descrito en este artículo.

- Iniciativas buscando aumentar la innovación a través del compromiso de corporaciones con startups, como el modelo Corporate Venture (Weiblein y Chesbrough, 2015). 
Sobre todo, el diferencial de la aplicación de estos modelos de innovación son los «resultados esperados». Anteriormente, los indicadores estaban muy enfocados en el resultado de los proyectos de I+D y en el registro de patentes, sin gran compromiso de llegar efectivamente al mercado con nuevos productos y servicios innovadores, dejando como legado para las organizaciones un stock de proyectos realizados y una colección de propiedades intelectuales. Cada vez más, los indicadores se enfocan en nuevos negocios innovadores con gran potencial de éxito, ya sea en el mercado actual o en nuevos mercados de las organizaciones que buscan la innovación.

Este artículo tiene como tema la gestión de la investigación, desarrollo e innovación $(I+D+i)$ y como objetivo describir la situación actual del proyecto MGPDI y del modelo MGPDI, un nuevo sistema de gestión de I+D+i que es aplicable a cualquier organización, independientemente del tamaño, tipo y actividad.

Este trabajo para 360: Revista de Ciencias de la Gestión -revista del Departamento Académico de Ciencias de la Gestión (DACG) de la PUCP_ es una versión revisada, reestructurada y modificada de la ponencia «MGPDI: Modelo de gestión de la I+D+i en las organizaciones» (Weber y otros, 2015a), presentada en el I Congreso Internacional de Gestión de la Innovación (realizado del 28 de septiembre al 2 de octubre de 2015 en la PUCP, Lima, Perú), que fue la primera comunicación de la puesta en marcha del nuevo modelo MGPDI.

Crear un nuevo modelo para mejorar continuamente los procesos de gestión de la I+D+i no es una tarea trivial. Hay dos grandes desafíos: un complejo desafío técnico y el desafío de conquistar mercados llenos de incertidumbres. Ambos requieren conocimiento multidisciplinar e implican largos plazos. Respecto a los riesgos, incluso aunque obtenga el éxito en la parte técnica, no hay garantía de que el nuevo modelo sea aceptado en el mercado.

Esta sección introductoria del artículo delimita el tema del proyecto MGPDI (innovación abierta con un enfoque de gestión de I+D+i en las organizaciones) y presenta el objetivo del artículo. En la sección 2, es descrito el nuevo modelo de proceso MGPDI. En las secciones 1 y 2 también se hace una breve revisión de la literatura. La sección 3 presenta la situación del proyecto y del modelo MGPDI, a diciembre de 2016. En la sección 4 se encuentran las conclusiones y consideraciones finales del trabajo.

\section{Modelo MGPDI}

El propósito del modelo de proceso MGPDI es contribuir efectivamente a promover la innovación y mejorar continuamente los procesos de gestión de la investigación, desarrollo e innovación en las organizaciones.

Este nuevo modelo de proceso para la gestión de la $1+D+i$ se ha creado desde el comienzo de 2015, bajo la coordinación de una asociación que apoya el desarrollo de software (SOFTSUL), teniendo en cuenta las lecciones aprendidas a partir del modelo MPS para la «Mejora de proceso de software» (Montoni, Rocha y Weber, 2009; Kalinowski y otros, 2015). El modelo MGPDI está siendo consolidado en Brasil y tiene gran potencial de ser replicado en países de América Latina y del Caribe, en portugués y español. 


\subsection{Componentes del Modelo MGPDI}

El gráfico 1 muestra los tres componentes del modelo MGPDI:

- El modelo de referencia MR-MGPDI (Process Reference Model o PRM), publicado en la Guía general del MGPDI, que se basa:

- En buenas prácticas de la innovación de la metodología MGPDI, existente desde 2008, a saber: Manual de Frascati (OECD, 2002), Manual de Oslo (OECD, 2005), Innovación abierta (Chesbrough, 2003), Golnnovate (Papageorge, 2004), TRIZ (Altshuller, 1999), gestión de riesgos (Ritter, 2008) y gestión del conocimiento.

- En directrices de la norma brasileña ABNT NBR 16501 (2011) y en requisitos de las normas españolas AENOR UNE 166001 (2006) y AENOR UNE 166002 (2014), todas para gestión de la innovación.

- El modelo de evaluación MA-MGPDI (Process Assessment Model o PAM), publicado en la Guía de evaluación del MGPDI, que se basa en la familia de normas internacionales ISO/IEC 33000 (2015), antigua ISO/IEC 15504, para evaluación de procesos (también son referencias: SOFTEX (2015), CMMI (2014) y CTI Renato Archer (2013).

- El modelo de negocio MN-MGPDI, con las reglas de negocio descritas en un documento de acceso restringido.

\section{Gráfico 1: Componentes del modelo MGPDI}

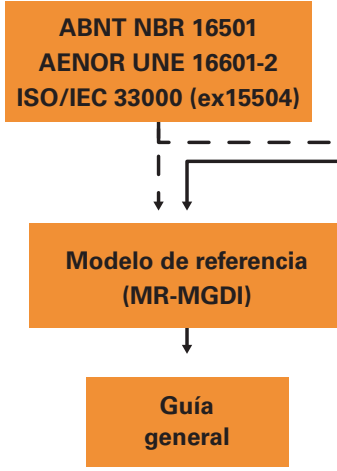

MGPDI

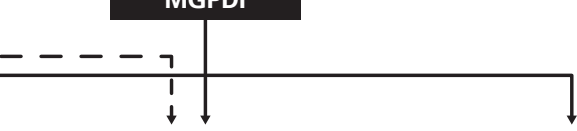

Modelo de evaluación

(MA-MGPDI)

$\downarrow$

Guía de evaluación
Modelo de negocio (MN-MGPDI)

$\downarrow$

Documento restringido

Fuente: Guía general y Guía de evaluación del MGPDI, 2016 (versión beta).

\subsection{Perfiles de proceso}

El gráfico 2 muestra que el modelo MGPDI está basado en los conceptos de mejora de procesos y determinación de la capacidad de procesos.

En dos dimensiones (dimensión de los procesos y dimensión de la calidad de los procesos), el modelo MGPDI comprende tres perfiles de proceso: perfil I, procesos ejecutados; perfil II, procesos gestionados; y perfil III, procesos definidos e 
institucionalizados. Este nuevo modelo de procesos fue diseñado de modo que la capacidad en el perfil I proporcione progresivamente la base para la mejora del nivel de madurez hacia los perfiles II y III, y así sucesivamente.

\section{Gráfico 2: Modelo MGPDI. Perfiles de proceso}

Modelo MGPDI para mejora de procesos y determinación de la capacidad

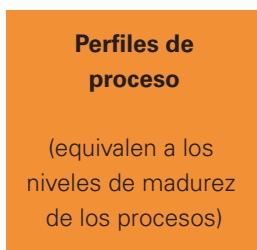

Perfil III - Procesos definidos e institucionalizados

Perfil II - Procesos gestionados

Perfil I - Procesos ejecutados

"n" Procesos de gestión de la PDI

Fuente: Guía general del MGPDI, 2016 (versión beta).

\subsection{Procesos y atributos de proceso}

La dimensión de procesos cuenta con trece procesos de innovación y de gestión de la I+D+i, para cada uno de los cuales el modelo de referencia MR-MGPDI define tanto un "propósito» específico como diversos "resultados esperados del proceso» (REP), no presentados en este trabajo debido a la limitación de espacio: 1) gobernanza (GOV), 2) gestión de la investigación (GPE), 3) gestión de la innovación (GIO), 4) gestión de proyectos de innovación (GPR), 5) gestión de indicadores de innovación (GIN), 6) gestión de riesgos (GRI), 7) estrategia de innovación (EIN), 8) gestión del ciclo de la innovación (GCI), 9) gestión de mudanzas (GMU), 10) gestión de configuración (GCO), 11) gestión de la calidad (GQU), 12) gestión de portafolio (GPO), y 13) gestión de la propiedad intelectual (GPI).

En la dimensión de la calidad de los procesos (capacidad), la madurez de estos es determinada por un conjunto de atributos de proceso (AP) que son propiedades mensurables de una característica de la calidad del proceso, conforme con lo dispuesto en la ISO/IEC 33020 (2015). Los atributos de proceso (AP) son acumulativos y requeridos para todos los procesos.

Para cada perfil, la tabla 1 presenta los respectivos procesos y atributos de proceso (Weber y otros, 2016):

Tabla 1: Modelo MGPDI (perfiles, procesos y atributos de proceso)

\begin{tabular}{l|l|l} 
perfil & proceso & atributo de proceso (AP) \\
\hline I Procesos ejecutados & $\begin{array}{l}\text { 1. Gobernanza (GOV) } \\
\text { 2. Gestión de la investigación (GPE) }\end{array}$ & AP 1.1: el proceso es ejecutado \\
& $\begin{array}{l}\text { 3. Gestión de la innovación (GIO) } \\
\text { 4. Gestión de proyectos de innovación (GPR) } \\
\text { 5. Gestión de indicadores de innovación (GIN) }\end{array}$ & Continúa
\end{tabular}


perfil
II Procesos gestionados

III Procesos definidos e institucionalizados
1. Gobernanza (GOV)

2. Gestión de la investigación (GPE)

3. Gestión de la innovación (GIO)

4. Gestión de proyectos de innovación (GPR)

5. Gestión de indicadores de innovación (GIN)

6. Gestión de riesgos (GRI)

7. Estrategia de innovación (EIN)

8. Gestión del ciclo de la innovación (GCl)

9. Gestión de cambios (GMU)

10. Gestión de configuración (GCO)

11. Gestión de calidad (GQU)

12. Gestión de portafolio (GPO)

1. Gobernanza (GOV)

2. Gestión de la investigación (GPE)

3. Gestión de la innovación (GIO)

4. Gestión de proyectos de innovación (GPR)

5. Gestión de indicadores de innovación (GIN)

6. Gestión de riesgos (GRI)

7. Estrategia de innovación (EIN)

8. Gestión del ciclo de la innovación (GCl)

9. Gestión de cambios (GMU)

10. Gestión de configuración (GCO)

11. Gestión de calidad (GQU)

12. Gestión de portafolio (GPO)

13. Gestión de la propiedad intelectual (GPI)
AP 1.1: el proceso es ejecutado AP 2.1-2.2: el desempeño del proceso y los productos de trabajo son gestionados

AP 1.1: el proceso es ejecutado AP 2.1-2.2: el desempeño del proceso y los productos de trabajo son gestionados AP 3.1-3.2: el proceso es definido e institucionalizado

Nota: en un futuro, considerando lo dispuesto en la ISO/IEC 33020 (2015), la capacidad del modelo MGPDI podrá ser aumentada con la adición del Perfil IV - Procesos en optimización, incluyendo los atributos de proceso "AP 4.1-4.2: análisis cuantitativo y control cuantitativo de proceso" y "AP 5.1-5.2: el proceso de innovación está todo implementado y en optimización».

\subsection{Implementación y evaluación del modelo MGPDI en organizaciones}

Un abordaje para promover la innovación y la gestión de la I+D+i en las organizaciones consiste en la adopción del «modelo de proceso» MGPDI. Esto es hecho en dos etapas:

a. Implementación del modelo MGPDI en la organización, según el modelo de referencia MR-MGPDI, basada en la Guía general del MGPDI y realizada con el apoyo de un consultor de implementación acreditado por la SOFTSUL. Dependiendo del perfil a ser implementado, normalmente esto dura de seis a doce meses.

b. Evaluación del modelo MGPDI en el perfil implementado en la organización y siguiendo el modelo de evaluación MA-MGPDI, basada en la Guía de evaluación del MGPDI y hecha por un equipo de evaluación acreditado por la SOFTSUL (evaluador líder y evaluadores adjuntos).

La regla básica es «nadie puede ser evaluador en la misma empresa/organización en que fue implementador y viceversa».

El gráfico 3 ilustra el flujo de la evaluación que comprende una evaluación (año 0), con dos seguimientos (años 1 y 2); o sea, 360 días y 720 días después de la fecha de 
conclusión de la evaluación. Después del vencimiento del plazo de vigencia de tres años de una evaluación vigente, es necesario realizar una nueva evaluación trienal (año 3).

\section{Gráfico 3: Flujo de la evaluación MGPDI}

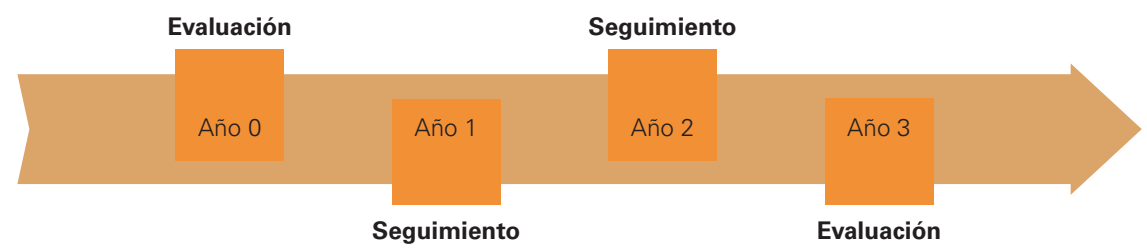

Fuente: Guía de evaluación del MGPDI, 2016 (versión beta).

El gráfico 4 muestra las cuatro fases de una evaluación trienal MGPDI, detallando las principales salidas de cada fase: 1) viabilizar la evaluación; 2) preevaluar (en principio, de modo remoto); 3) visitar la unidad organizacional (UO); y 4) encerrar la evaluación.

Los seguimientos anuales tienen como objetivo motivar y garantizar que la UO mantenga durante cada trienio el perfil de proceso obtenido en la evaluación MGPDI. En principio, estos dos seguimientos son hechos remotamente, con envolvimiento del evaluador líder, del representante de la SOFTSUL, del promotor de la evaluación y del punto de contacto en la UO con evaluación MGPDI vigente. Estos seguimientos anuales comprenden dos fases, con las respectivas actividades y salidas: fase 1, planificar seguimientos; y fase 2, ejecutar seguimientos.

\section{Gráfico 4: Fases de la evaluación trienal MGPDI}

\begin{tabular}{|c|c|c|c|}
\hline $\begin{array}{l}\text { VIABILIZAR LA } \\
\text { EVALUACIÓN }\end{array}$ & PREEVALUAR & $\begin{array}{l}\text { VISITAR LA UNIDAD } \\
\text { ORGANIZACIONAL }\end{array}$ & $\begin{array}{l}\text { ENCERRAR LA } \\
\text { EVALUACIÓN }\end{array}$ \\
\hline Salidas: & Salidas: & Salidas: & Salidas: \\
\hline $\begin{array}{l}\text { - Comunicado de la } \\
\text { evaluación }\end{array}$ & $\begin{array}{l}\text { Artefactos } \\
\text { prevalidados }\end{array}$ & $\begin{array}{l}\text { Entrevistas } \\
\text { realizadas }\end{array}$ & $\begin{array}{l}\text { Evaluación } \\
\text { auditada }\end{array}$ \\
\hline - Infraestructura & - Plan revisado & - Determinación del & - Evaluación \\
\hline - Reunión inicial & & nivel de madurez & publicada \\
\hline $\begin{array}{l}\text { Planificación de la } \\
\text { evaluación }\end{array}$ & & & \\
\hline
\end{tabular}

Fuente: Guía de evaluación del MGPDI, 2016 (versión beta).

\section{Situación actual del proyecto y del modelo MGPDI}

Esta sección presenta la situación actual, a diciembre de 2016, tanto del proyecto como del modelo MGPDI.

\subsection{Situación actual del proyecto MGPDI (diciembre, 2016)}

Los principales resultados obtenidos en 2015-2016 en el proyecto MGPDI fueron los siguientes:

a. Gestión del proyecto dirigida por un coordinador ejecutivo, basada en la metodología del marco lógico (Logical Framework Approach o LFA). 
b. Creación de la Unidad de Ejecución del Proyecto UEP-MGPDI, con cuatro miembros que realizan mensualmente reuniones vía Skype de seguimiento del proyecto.

c. Creación del Consejo de Gestión del Proyecto CGP-MGPDI, compuesto por las partes interesadas (stakeholders) que se reúnen semestralmente para apoyar a la SOFTSUL en la planificación de las actividades anuales del MGPDI y en el seguimiento de la ejecución de esas actividades.

d. Creación del equipo técnico del modelo ETM-MGPDI, compuesto por miembros invitados por la SOFTSUL escogidos entre profesionales con experiencia en gestión de la innovación y en mejora de procesos, para apoyar el proyecto MGPDI en la:

- Definición del modelo de referencia MR-MGPDI y del modelo de evaluación MA-MGPDI.

- Creación de las guías del MGPDI (inicialmente, Guía general y Guía de evaluación).

- Capacitación de personas en el modelo MGPDI por medio de cursos, pruebas y workshops.

- Sumisión de artículos en simposios y revistas en portugués, español e inglés.

- Internacionalización del modelo de proceso MGPDI.

\subsection{Situación actual del modelo MGPDI (diciembre, 2016)}

Los principales resultados obtenidos en 2015-2016 con el modelo MGPDI fueron los siguientes:

a. Validación de dos guías MGPDI (Guía general y Guía de evaluación, versiones beta), para publicación hasta diciembre de 2016 (en portugués y español), disponible en <http://softsul.org.br/mgpdi/?lang=pb>.

b. Calificación y acreditación, hecha por la SOFTSUL, de los primeros implementadores, evaluadores (líderes y adjuntos), auditores de evaluación e instructores MGPDI.

c. Constitución de la red de colaboradores de la línea de frente MGPDI (implementadores, evaluadores y auditores de evaluación, instructores y organizadores de grupos de empresas) según el concepto del triángulo del servicio (Teboul, 2008).

d. Liberación, hecha por la SOFTSUL, del acceso a dos herramientas de software en ambiente colaborativo:

- Sistema de Gestión de la Investigación, Desarrollo e Innovación (SGPDI) para apoyar a la organización en la implementación y en la evaluación MGPDI.

- Appraisal Assistant (AA) para dar soporte a la evaluación MGPDI en las organizaciones. 
e. Hecho relevante: exitosas implementaciones y evaluaciones piloto del modelo MGPDI en tres organizaciones de la región Sur de Brasil, como es relatado en Weber y otros (2016):

- $\quad$ Empresa A (Schalter Eletrônica LTDA, de Porto Alegre-RS) ${ }^{12}$ :

- Implementación piloto MGPDI de noviembre de 2015 a junio de 2016.

- Evaluación piloto MGPDI concluida el 9 de noviembre de 2016.

- $\quad$ Empresa B (Kenta Informática SA, de Porto Alegre-RS) ${ }^{13}$ :

- Implementación piloto MGPDI de noviembre de 2015 a junio de 2016.

- Evaluación piloto MGPDI: «preevaluar» el 22 de noviembre y «Visitar UO», prevista para el 9 de diciembre de 2016

- $\quad$ Empresa C (Uninfo Sistemas LTDA - ME, de Chapecó-SC) ${ }^{14}$ :

- Implementación piloto MGPDI de diciembre de 2015 a junio de 2016.

- Evaluación piloto MGPDI concluida el 31 de octubre de 2016.

\section{Conclusiones y consideraciones finales}

Creemos haber logrado el objetivo de este artículo; o sea, describir el nuevo modelo de proceso MGPDI y la situación actual a diciembre de 2016 del proyecto y del modelo MGPDI, especialmente la realización de implementaciones y evaluaciones piloto en tres empresas brasileñas.

También llegamos a la conclusión de que el modelo MGPDI, escalonado por perfiles de proceso, fue adaptado a su objetivo que es contribuir de manera efectiva a la promoción de la innovación y la mejora de la gestión de la I+D+i en las organizaciones.

En el periodo 2015-2016, este proyecto ha construido dos activos muy importantes:

- En la parte técnica, la metodología MGPDI ya existente fue transformada efectivamente en el nuevo modelo de proceso MGPDI.

- Respecto al mercado, tres implementaciones y evaluaciones piloto en Brasil fueron exitosas.

A largo plazo, el proyecto MGPDI tiene dos metas (desafíos permanentes):

- Una meta técnica, que busca la mejora continua del modelo MGPDI.

- Una meta de mercado, direccionada a una creciente difusión del modelo MGPDI en Brasil y su replicación en otros países de América Latina y del Caribe, en portugués y español. Según la demanda del mercado, también podrá ser replicado en inglés.

12. Consultar: <http://schalter.com.br>

13. Consultar: <https://www.kenta.com.br $>$

14. Consultar: <http://uninfosistemas.com.br $>$. 
Una sugerencia para trabajos futuros, lo que requeriría tiempo y metodologías apropiadas, es comparar el modelo MGPDI con otros sistemas de gestión de la innovación. En este sentido, se recomienda que la comparación tenga en cuenta aspectos técnicos y también aspectos de mercado (Go2Market).

Así, con este modelo de proceso MGPDI, esperamos contribuir a la promoción de la innovación abierta y a la difusión de las buenas prácticas para mejorar la gestión de I+D+i con el fin de aumentar la competitividad de cualquier organización independientemente de su tamaño, tipo y actividad.

También esperamos que este artículo pueda contribuir a una mejor comprensión de la gestión de la innovación abierta en las organizaciones, ya sea para profesionales, instructores, implementadores y evaluadores de sistemas o modelos de gestión de la I+D+i, o, en la academia, para estudiantes, profesores e investigadores interesados en este tema.

Por último, pero no menos importante, cabe señalar que la innovación abierta es uno de los perfiles profesionales prometedores : «En el mercado competitivo del futuro, la gestión de la innovación y la gestión de la investigación y desarrollo serán parte de la vida cotidiana de las industrias [...]» (Valença, 2016). 


\section{ABNT NBR 16501}

2011

Diretrizes para sistemas de gestão da pesquisa, do desenvolvimento e da inovação (PDI). Norma Brasileira.

\section{AENOR UNE 166001}

2006

Gestión de la 1+D+i: Requisitos de un

proyecto de $1+D+i$. Norma española.

\section{AENOR UNE 166002}

2014 Gestión de la 1+D+i: Requisitos del sistema de gestión de la l+D+i. Norma española.

\section{Altshuller, Genrich}

1999

[1969] Innovation Center.

\section{Associação para Promoção da Excelência do Software} Brasileiro (SOFTEX) MPS.BR - Guia de Avaliação. Disponible en: <http://www.softex.br/mpsbr>.

\section{Centro de Tecnologia da} Informação Renato Archer (CTI Renato Archer)

Método de Avaliação CERTICS: versão 1.1.

Disponible en: <http://www.certics.cti. gov.br>.

\section{Belussi, Fiorenza; Alessia}

\section{Sammarra \& Silvia Rita Sedita}

Regional Innovation System': A focus on firms' innovation strategies in the Emilia

Romagna life science industry». Research Policy, 39 (6), pp. 710-721. Disponible en: $<$ http://economia.unipd.it/sites/decon. unipd.it/files/20100113.pdf>; <https://doi. org/10.1016/i.respol.2010.01.014>.

\section{Charan, Ram}

2015

The Attacker's Advantage: Turning

Uncertainty into Breakthrough

Opportunities. Nueva York: Publish Affairs.

\section{Chesbrough, Henry}

2003

Open Innovation: The New Imperative for Creating and Profiting from Technology. Boston: HBS Press.

\section{CMMI Product Team (CMMI)}

2014

Standard CMMI Appraisal Method for

Process Improvement (SCAMPISM)

Version 1.3b: Method Definition Document.

Pittsburgh, PA: Software Engineering

Institute, Carnegie Mellon University.

\section{Ferreira, Rafael \& Paulo}

\section{Cauchick Miguel}

2013

«Análise Comparativa sobre Processos de Inovação da Literatura com a Norma Brasileira de Gestão da Inovação». Exacta, 11 (3), pp. 285-297.

\section{Hansen, Morten \& Julian}

\section{Birkinshaw}

"The innovation value chain». Harvard Business Reviev, 85 (6), pp. 121-130.

\section{ISO/IEC 33000}

Information technology - Process assessment (new family ISO/IEC 33000 updates ISO/IEC 15504 series).

\section{Kalinowski, Marcos y otros}

«Software Process Improvement Results in Brazil Based on the MPS-SW Model». Software Quality Professional Magazine, 17 (4), pp. 15-28. Disponible en: <http:// asq.org/pub/sqp/past/vol17_issue4/index. $\underline{\mathrm{html}}>$. 


\section{bibliografía}

Montoni, Mariano Angel, Ana

Regina Rocha \& Kival Chaves

Weber

2009

"MPS.BR: A successful program for

software process improvement in Brazil».

Software Process: Improvement and

Practice, 14 (5), pp. 289-300. Disponible

en: <https://doi.org/10.1002/spip.428>

\section{Organization for Economic}

Co-operation and Development (OECD)

Frascati Manual. Proposed Standard

Practice for Surveys on Research and

Experimental Development (sexta edición).

París: OECD. Disponible en: <http://www.

tubitak.gov.tr/tubitak_content_files/BTYPD/

kilavuzlar/Frascati.pdf $>$.

\section{Organization for Economic}

Co-operation and Development (OECD)

Oslo Manual. Guidelines for Collecting

and Interpreting Innovation Data (tercera

edición). París: OECD. Disponible en:

$<$ http://www.oecd-ilibrary.org/docserver/

download/9205111e.pdf?expires $=1491$

$\underline{795781 \& i d=i d \& a c c n a m e=\text { guest\& }}$

checksum $=038$ DF592FE65AEBAA94005

E9F258DA4A > ; <https://doi.

org/10.1787/9789264013100-en>.

\section{Papageorge, Andrew}

2004 Golnnovate! A Practical Guide to Swift, Continual and Effective Innovation. San

Diego, CA: Golnnovate! Publishing.

\section{Ranga, Maringa \& Henry \\ Etzkowitz}

2013

«Triple helix systems: An analytical

framework for innovation policy and

practice in the knowledge society".

Industry and Higher Education, 27 (3), pp.

237-262. Disponible en: <http://triplehelix.

stanford.edu/images/Triple Helix

Systems.pdf > ; <https://doi.org/10.5367/

ihe.2013.0165 $>$

\section{Ritter, Donna}

2008

Risk Breakdown Structure (RBS).

Disponible en: < http://certifedpmp.

wordpress.com/2008/10/11/risk-

breakdown-structure-rbs/>.

\section{Teboul, James}

Serviços em Cena: o diferencial que agrega valor ao seu negócio. Brasilia: ELSEVIER /

INSEAD / CAMPUS / CNI-IEL/NC.

\section{Valença, Raquel}

2016

«Pesquisa Multissetorial da Federação das Indústrias do Estado do Paraná (FIEP).

Observatórios Sesi/Senai/FIEP». Gazeta do Povo, 12/11/2016, p. 32.

\section{Weber, Kival Chaves \& otros}

«MGPDI: Modelo de gestión de la

I+D+i en las organizaciones».

I Congreso Internacional de Gestión de la Innovación, 28 septiembre-2 octubre 2015, PUCP, Lima, Perú. Disponible en: <http://congreso.pucp.edu.pe/ gestion-innovacion/ediciones-anteriores/icongreso-internacional-ciencias-la-gestioninnovacion/?seccion=resumen $>$. 